\title{
Intracochlear electrode array position and cochlear implant outcomes using the nucleus slim modiolar electrode and the extended round window approach: a follow-up study
}

\author{
Tim M. Klabbers ${ }^{1,3}$ (10 $\cdot$ Floris Heutink $^{1,3} \cdot$ Wendy J. Huinck ${ }^{1,3} \cdot$ Willem-Jan van der Woude $^{2} \cdot$ Berit M. Verbist $^{2,4}$. \\ Emmanuel A. M. Mylanus ${ }^{1,3}$
}

Received: 15 November 2021 / Accepted: 28 December 2021 / Published online: 18 January 2022

(c) The Author(s) 2022

\begin{abstract}
Purpose The aim of this study was to evaluate the intracochlear position of the Slim Modiolar Electrode (SME) after insertion via the extended Round Window (eRW) approach, and to correlate this with residual hearing preservation and speech perception outcomes.

Methods Twenty-three adult participants, consecutively implanted with the SME via the eRW approach, were included in this prospective, single-center, observational study. Electrode position was evaluated intra-operatively using X-ray fluoroscopy and TIM measurement, and post-operatively using ultra-high resolution CT. Residual hearing [threshold shift in PTA between pre- and post-operative measurement, relative hearing preservation (RHP\%)] and speech perception were evaluated at 2 and 12 months after surgery.

Results In each of the 23 participants, complete scala tympani positioning of the electrode array could be achieved. In one participant, an initial tip fold-over was corrected during surgery. Average age at implantation was 63.3 years (SD 13.3, range 28-76) and mean preoperative residual hearing was $81.5 \mathrm{~dB}$. The average post-operative PTA threshold shift was $16.2 \mathrm{~dB}$ (SD 10.8) at 2 months post-operatively, corresponding with a RHP\% score of 44\% (SD 34.9). At 12 months, the average RHP\% score decreased to $37 \%$. Postoperative phoneme scores improved from $27.1 \%$ preoperatively, to $72.1 \%$ and $82.1 \%$ at 2 and 12 months after surgery, respectively.

Conclusion Use of the eRW approach results in an increased likelihood of complete scala tympani insertion when inserting the SME, with subsequent excellent levels of speech perception. However, residual hearing preservation was found to be moderate, possibly as a result of the extended round window approach, emphasizing that it is not an all-purpose approach for inserting this particular electrode array.
\end{abstract}

Keywords Cochlear implantation $\cdot$ Residual hearing $\cdot$ Slim modiolar electrode $\cdot$ Extended round window approach

Tim M. Klabbers

tim.klabbers@radboudumc.nl

1 Department of Otorhinolaryngology and Head and Neck Surgery, Radboud University Medical Center, Philips Van Leydenlaan 15, 6525 EX Nijmegen, The Netherlands

2 Department of Radiology, Radboud University Medical Center, Nijmegen, The Netherlands

3 Donders Institute for Brain, Cognition and Behaviour, Nijmegen, The Netherlands

4 Department of Radiology, Leiden University Medical Center, Leiden, The Netherlands

\section{Introduction}

With the expansion of the indication criteria for cochlear implantation $(\mathrm{CI})$, preservation of residual hearing has become an important objective [1]. An increasing body of evidence suggests that preservation of residual hearing and overall audiological outcomes are directly influenced by the occurrence of insertional trauma during cochlear implantation [2]. Mechanical trauma during electrode insertion can include osseous spiral lamina fracture, lateral wall or modiolar injury, basilar membrane disruption and electrode translocation from the scala tympani (ST) to the scala vestibuli (SV) [3]. In some cases, the electrode is even inserted directly into the SV due to misplacement of 
the cochleostomy [4]. Several studies conclude that complete placement of the active electrode within the ST is associated with better performance with the CI, compared to partial or complete SV placement [5-7]. This has led to an increased interest in the surgical approach to the cochlea, hypo-traumatic surgical techniques and the development of hypo-traumatic electrode arrays.

Currently available CI electrode arrays can be categorized into two main types: 'straight' or 'pre-curved'. Straight electrode arrays essentially follow the lateral wall of the cochlea once implanted. Pre-curved, or (peri) modiolar electrode arrays on the other hand, are designed to curl around the medial wall and position the electrode contacts in proximity to the modiolus and spiral ganglion cells, thus leading to a reduced spread of excitation, lower stimulation thresholds and improved stimulation specificity [8-11]. However, conventional perimodiolar electrodes are known to lead to a higher rate of translocation to the scala vestibuli, resulting in poorer speech perception outcomes [12]. Advancements in the design of pre-curved electrode arrays led to the development of the slim modiolar electrode (SME) in 2016; an electrode that is $60 \%$ thinner and more flexible than the previous generation of perimodiolar electrodes produced by the same manufacturer [13]. This electrode is designed to minimize trauma during insertion and to preserve residual hearing.

However, a recent study by Heutink et al. in 23 adult participants implanted with a SME via the cochleostomy (CS) approach revealed a translocation from the scala tympani to the scala vestibuli in $36.4 \%$ of the participants [4]. Translocation was subsequently observed to lead to significantly lower levels of residual hearing preservation (RHP) (19.7\% compared to $77.2 \%$ in participants with full ST placement) and lower speech recognition scores.

Several studies, therefore, advocate the use of either the round window (RW) or extended round window (eRW) insertion approach when using the SME, as this has been shown to decrease the probability of scalar excursion and thus improve audiological outcomes and hearing preservation $[2,12,14]$. These two approaches are, however, often grouped together and studies solely investigating outcomes of implantations of the SME via the extended round window approach are scarce. The primary objective of this prospective study was to evaluate the intracochlear position (i.e. occurrence of translocation from scala tympani to scala vestibuli, presence of a tip fold-over) of the SME after implantation using the extended round window technique and to correlate this to post-operative audiological outcomes such as hearing preservation and speech perception. Additionally, we aim to compare these findings to previously published results obtained with the SME using different surgical approaches.

\section{Methods}

\section{Study design}

This was a prospective, single-center observational study in twenty-three adult patients consecutively implanted with the Slim Modiolar Electrode (SME; Nucleus ${ }^{\circledR}$ CI532/CI632; Cochlear Ltd., Sydney, Australia) using the extended Round Window (eRW) approach. Participants were included between July 2019 and July 2020. Exclusion criteria were (1) pre-lingual onset of deafness, (2) anatomical variations of the cochlea possibly influencing electrode insertion and (3) inability to undergo CT-scanning. Preoperatively, demographic data including the history of hearing loss and audiometry were collected. X-ray fluoroscopy and trans-impedance matrix (TIM) measurements were performed intra-operatively to rule out tip fold-overs. Electrode position was evaluated 1 week post-implantation using an ultra-high resolution computed tomography (UHRCT) scan. Residual hearing was evaluated routinely 2 months post-operatively, whilst speech perception was measured routinely 2 and 12 months post-operatively. Several participants included in the current study were also reported on in a proof-of-concept article by Klabbers et al. on the use of TIM measurements for initial tip fold-over detection. However, no clinical outcomes were evaluated in the previous study. All procedures performed in this study were in accordance with the 1964 Helsinki Declaration and its later amendments [15]. Institutional Review Board approval was obtained and all patients signed informed consent forms. The study was registered in the Netherlands Trial Register (trial number NL8290).

\section{Surgical procedure}

Surgery was performed by three experienced CI-surgeons, and the standard mastoidectomy and facial recess approach was used to gain access to the cochlea in all cases. The bony overhang of the round window niche was carefully removed and the scala tympani was opened using the extended round window technique, in which a so-called round window margin cochleostomy is created by carefully drilling the anterior-inferior bony margin and crista fenestrae (Fig. 1). All 23 patients were implanted with the pre-curved, slim modiolar electrode (SME). The SME has an active component with a length of $14 \mathrm{~mm}$ and a diameter of $0.35 \mathrm{~mm} \times 0.4 \mathrm{~mm}$ apically and $0.45 \mathrm{~mm} \times 0.5 \mathrm{~mm}$ basally. It is loaded into a protective plastic sheath of $5 \mathrm{~mm}$ in length and $0.77 \mathrm{~mm}$ in diameter and is inserted into the cochlea until the sheath stopper reaches the round window opening. Using forceps, the SME array is then slowly advanced until fully inserted (approx.120 s), after which 


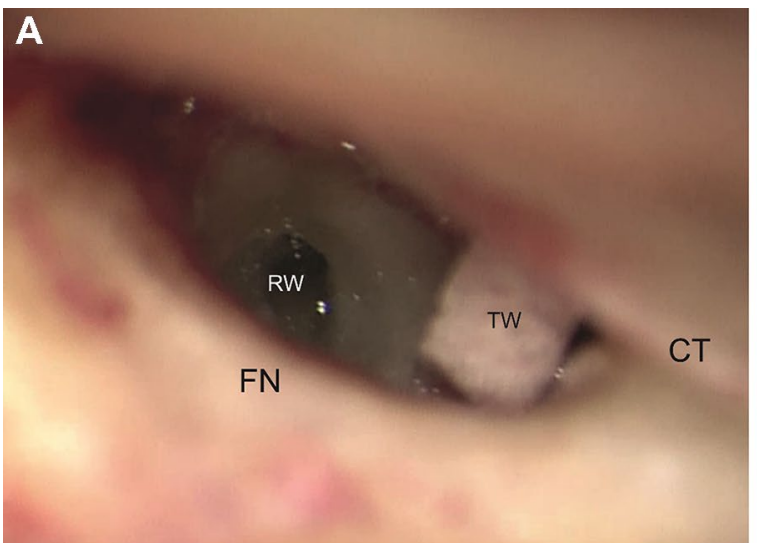

Fig. 1 Intraoperative view of the round window of a right ear through the facial recess after completion of the cortical mastoidectomy and posterior tympanotomy. A Before antero-inferior drilling and $\mathbf{B}$ after

the sheath is carefully retracted and removed. Depending on the surgeons' preferences, the extended round window opening was sealed with periosteum or fascia and fibrin glue. During surgery, patients were given a single dose of $1.8 \mathrm{mg} / \mathrm{kg}$ intravenous methylprednisolone.

\section{Evaluation of intracochlear electrode position}

Directly following electrode insertion and packing, TIM measurements and fluoroscopy were performed to rule out electrode array tip fold-over. In the event of a tip fold-over, the array was retracted and repositioned in the same surgical setting. One week post-operatively, electrode array position was evaluated using a UHRCT-scan (Aquilion Precision, Canon Medical Systems, Otawara, Japan). For scalar position analysis, the UHRCT-images were reconstructed using multiplanar reconstruction (MPR) and filtered back projection (bone filter kernel FC81), with a field of view of $90 \mathrm{~mm}, 1024 \times 1024$ matrix and $0.25 \mathrm{~mm}$ slice thickness. Mid-modiolar sections of the reconstructed UHRCT-scan images were evaluated by an experienced head and neck radiologist (Fig. 2). Each of the twenty-two electrode contacts was individually assessed and scored as either located in the ST or SV.

\section{Audiological evaluation}

Unaided pure tone audiometry at 125, 250, 500, 1000 and $2000 \mathrm{~Hz}$ was performed pre- and post-operatively for both ears using headphones in a soundproof booth following standard audiometry and masking protocol at our center. The low frequency pure tone average (LFPTA), defined as the average threshold at 250,500 and $1000 \mathrm{~Hz}$, was calculated for each patient. If a patient failed to respond to the

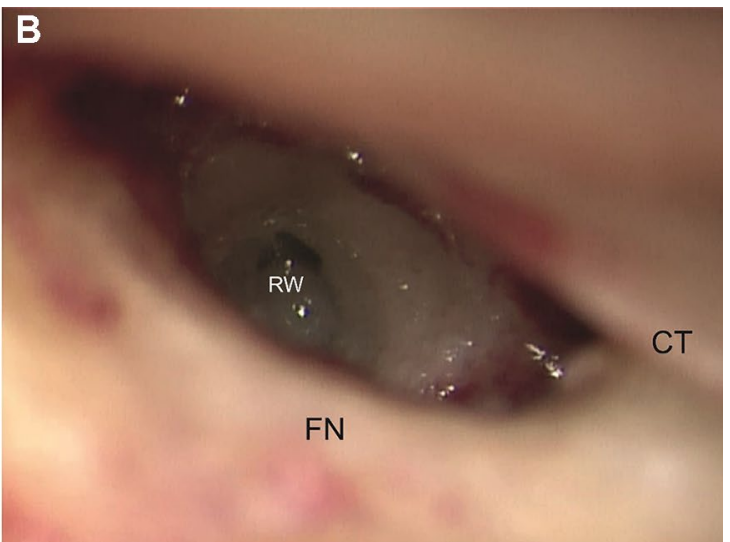

antero-inferior drilling. $F N$ facial nerve, $R W$ round window, $T W$ tissue wipe, $C T$ chorda tympani nerve

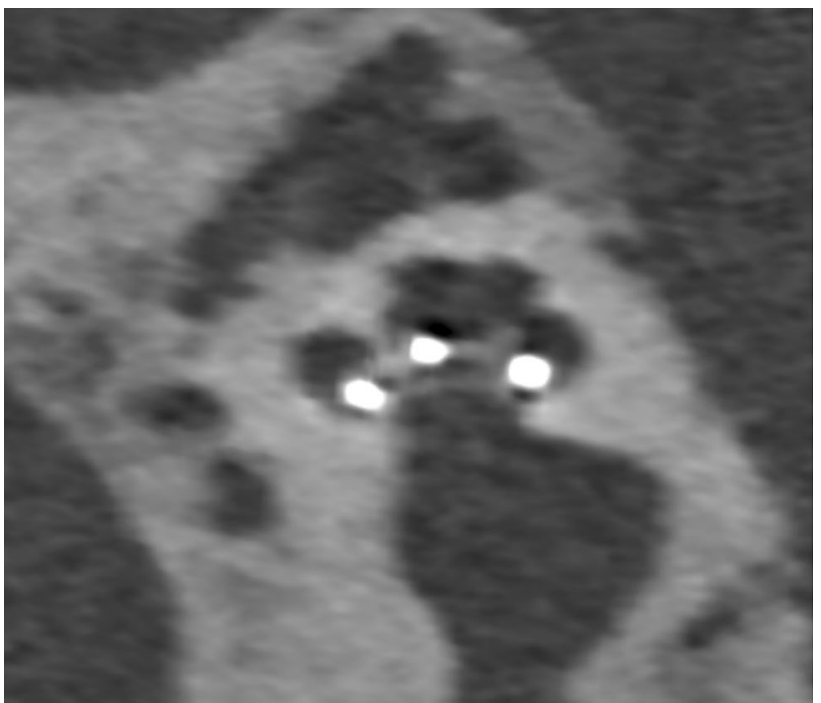

Fig. 2 Mid-modiolar section of the post-implantation UHRCT of participant \#12 indicating scala tympani placement of the electrode array

stimulus at a certain frequency, the maximum stimulation level (MSL) was recorded (as per Skarzynski et al. [16]). For the frequencies evaluated in this study, MSLs were $90 \mathrm{~dB}, 105 \mathrm{~dB}, 110 \mathrm{~dB}, 120 \mathrm{~dB}$ and $120 \mathrm{~dB}$, respectively. Failure to respond to two or more of the frequencies for calculating the LFPTA was recorded as "non-measurable hearing" (NMH). In accordance with the consensus paper by the HEARRING group, residual hearing preservation (RHP) was calculated using the hearing preservation clas-

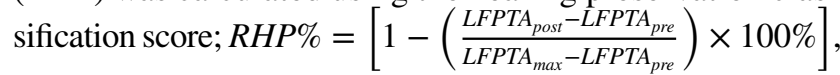

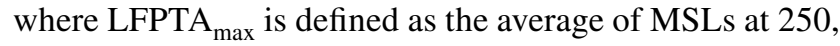
500 and $1000 \mathrm{~Hz}$ [16]. 
RHP percentages for each participant were categorized as either (1) complete preservation $(>75 \%)$, (2) partial preservation $(25-75 \%)$ or (3) minimal preservation (0-25\%). Additionally, mean threshold shifts in LFPTA between pre- and post-operative testing were evaluated. If necessary, these values were corrected for the natural progression of hearing loss, as previously described by Snels et al. ( $\triangle$ LFPTACIear $-\Delta$ LFPTAcontralateral(CL)ear) [17].

Speech perception in quiet was evaluated routinely at 2 and 12 months post-operatively. The standard test to assess speech perception in the Netherlands is issued by the Dutch Society of Audiology (NVA) and consists of a list of phonetically balanced, monosyllabic consonant-vowelconsonant (CVC) words presented at $65 \mathrm{~dB}$ SPL through a speaker which is placed in front of the participant in a soundproofed booth. The average percentage of three CVC lists was calculated.

\section{Statistical analysis}

Statistical evaluation was performed using SPSS (version 25, Armonk, New York). Continuous data are reported as mean values with standard deviations and comparisons between the audiological outcomes of the current and previous study were performed using independent samples $T$ tests. $P$ values $<0.05$ were deemed statistically significant.

\section{Results}

\section{Demographics}

Twenty-three patients were consecutively implanted with the SME at our center. Of these patients, 11 (47.8\%) were male and $12(52.2 \%)$ female. Fourteen patients $(60.9 \%)$ were implanted in the right ear, whilst nine (39.1\%) were implanted in the left ear. The average age at implantation was 63.3 years (SD 13.3; range 28-76) and participants had a mean preoperative residual hearing (LFPTA) of $81.5 \mathrm{~dB}$ (SD 11.9).

\section{Scalar position and tip fold-over evaluation}

Upon post-operative UHRCT scan evaluation, all of the electrode contacts for each of the 23 patients included in this study were found to be located fully within the scala tympani: no translocation had occurred. Intraoperative fluoroscopy and TIM measurement revealed a tip fold-over in 1 of the 23 patients $(4.3 \%)$. In this patient, subsequent repositioning of the electrode array in the same surgical setting led to a satisfactory intracochlear position as confirmed by repeated fluoroscopy and TIM measurements, as well as a complete
ST positioning of the array as confirmed with UHRCT 1 week post-operatively. Postoperative UHRCT evaluation did not reveal a tip fold-over in any of the other participants.

\section{Audiometry}

Postoperative residual hearing thresholds and speech perception scores for all twenty-three participants are presented in Table 1. The mean threshold shift in LFPTA at activation across all participants without tip fold-over was $16.2 \mathrm{~dB}$ (SD 10.8), whilst the mean RHP\% was 43.9\% (SD 34.8) (Table 2). The mean threshold shift in LFPTA in the contralateral, non-implanted ear was $0.1 \mathrm{~dB}$ and, therefore, no correction for the natural progression of hearing loss was deemed necessary.

According to the HEARRING classification, 7 out of $22(31.8 \%)$ participants had complete hearing preservation (RHP $>75 \%)$ at activation, whilst 6 out of $22(27.3 \%)$ had partial preservation (RHP 25-75\%). 9 out of 22 participants (40.9\%) had minimal or no preservation of residual hearing (RHP $<25 \%$ ). Of the 7 participants who had a preoperative LFPTA of less than $80 \mathrm{~dB}$, only 3 maintained a LFPTA lower than $80 \mathrm{~dB}$ at activation.

Long-term residual hearing preservation (RHP\%) was evaluated at 12 months post-operatively and was found to have deteriorated by an average of $7 \%$ (from 44 to $37 \%$ ), indicating a progression of residual hearing loss over time.

The mean post-operative CVC-phoneme score at 2 months was $72.1 \%$, and this further improved to $81.1 \%$ at 12 months (Table 3).

The post-operative threshold shift in LFPTA in the participant with an intraoperative tip fold-over was $10 \mathrm{~dB}$, whilst the relative hearing preservation score according to the HEARRING classification was $0 \%$. Speech perception scores at 2 and 12 months were $93 \%$ and $93 \%$, respectively.

\section{Discussion}

This study was initiated to evaluate the intracochlear electrode position and audiological outcomes of patients with the slim modiolar electrode, implanted exclusively using the extended round window approach. This is currently the approach of choice at our tertiary referral hospital for inserting this particular electrode, as a previous study (conducted at our center) revealed translocations of the SME from scala tympani to scala vestibuli in $36 \%$ of the patients implanted via cochleostomy. The electrode arrays of all participants included in the current study were found to be located fully within the scala tympani following extended round window insertion, resulting in excellent levels of speech perception scores. 


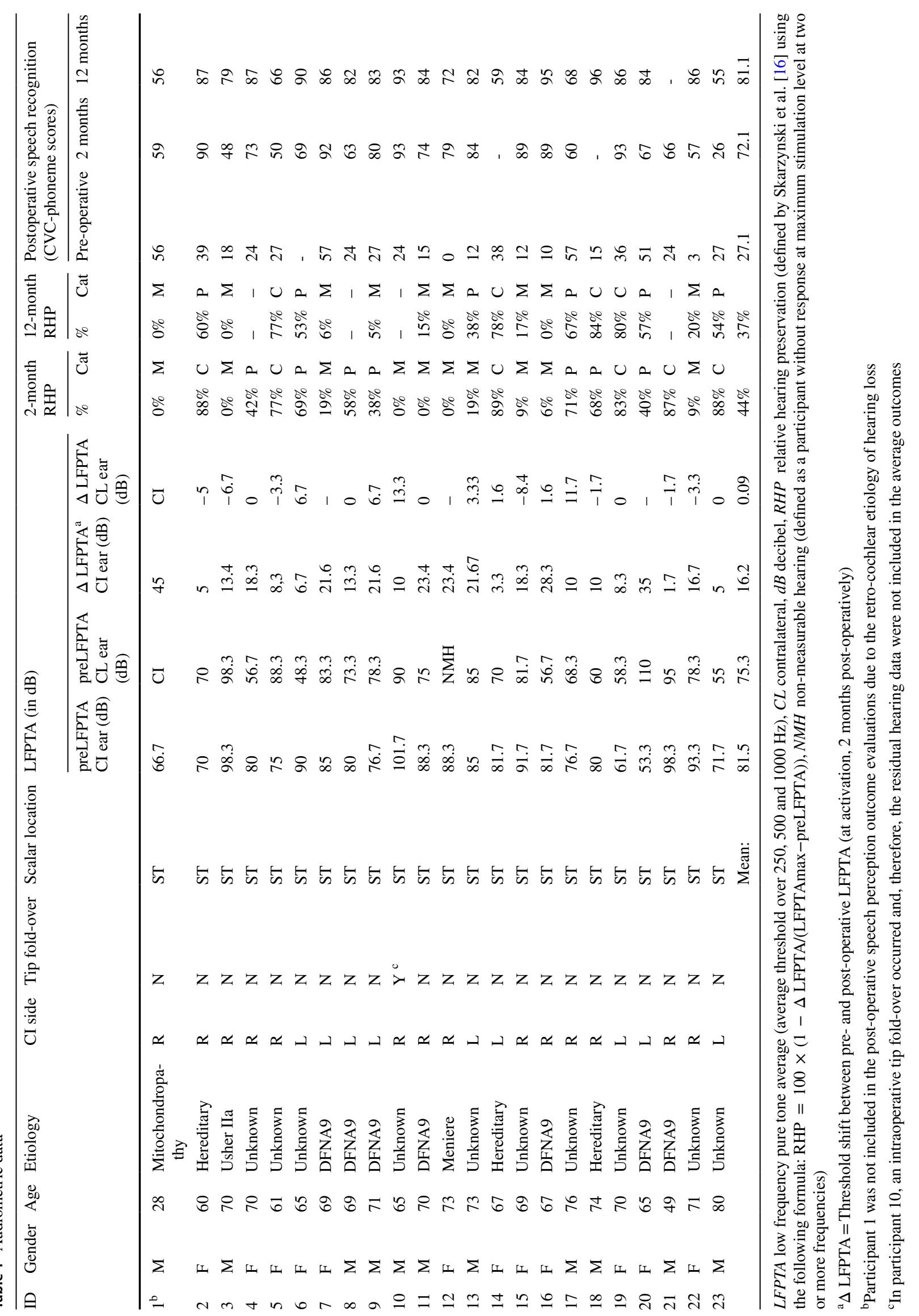


Table 2 Residual hearing outcomes obtained in the current study compared to the results obtained in the previous study by Heutink et al. (CS), as well as specifically for participants in whom the elec-

\begin{tabular}{|c|c|c|c|c|c|c|c|c|c|c|c|}
\hline & \multicolumn{2}{|c|}{$\begin{array}{l}\text { eRW (current } \\
\text { study) }\end{array}$} & \multicolumn{3}{|c|}{ CS (Heutink et al.) } & \multicolumn{3}{|c|}{ CS-ST (Heutink et al.) } & \multicolumn{3}{|c|}{$\begin{array}{l}\text { CS-Translocation (Heutink } \\
\text { et al.) }\end{array}$} \\
\hline & Mean (SD) & $N$ & Mean (SD) & $N$ & $p$ value & Mean (SD) & $N$ & $p$ value & Mean (SD) & $N$ & $p$ value \\
\hline Preoperative LFPTA $^{1}$ in $\mathrm{dB}$ & $81.5(11.9)$ & 23 & $92.2(13.3)$ & 19 & 0.009 & $94.9(10.7)$ & 12 & 0.003 & $87.1(19)$ & 7 & 0.296 \\
\hline $\begin{array}{l}\text { Postoperative threshold shift } \\
\text { in LFPTA in } \mathrm{dB}\end{array}$ & $16.2(10.8)$ & 22 & $9.2(9.2)$ & 17 & 0.039 & $4.4(5.3)$ & 11 & 0.002 & $17.9(9)$ & 6 & 0.723 \\
\hline RHP\% as per Skarzynski ${ }^{2}$ & $43.9(34.8)$ & 22 & $56.9(46.3)$ & 17 & 0.269 & $77.2(45)$ & 11 & 0.022 & $19.8(16)$ & 6 & 0.042 \\
\hline
\end{tabular}

${ }^{\mathrm{a}}$ One `participant (\#10) was excluded from hearing outcome analysis due to a tip fold-over

${ }^{1}$ Low frequency pure tone average: average threshold for 250,500 and $1000 \mathrm{~Hz}$

${ }^{2}$ Residual hearing preservation, RHP $\%=\left[1-\left(\frac{\text { LFPTA }_{\text {post }}-\text { LFPTA }_{\text {pre }}}{\text { LFPTA }_{\text {max }}-\text { LFPTA }_{\text {pre }}}\right) \times 100 \%\right]$

Implantation of the SME via the extended round window approach, therefore, seems to invariably lead to correct positioning within the scala tympani. These results are an evident improvement compared to previously reported implantations via a cochleostomy approach at our center, and generally comparable to what is reported in the literature, although studies adequately reporting on the CT-evaluated scalar position by surgical approach for the SME are still relatively scarce. The studies in which this was determined using high-resolution imaging result in a combined average translocation risk of $4.8 \%(22 / 461)$ [ $4,18-24]$. Of these aggregated 461 participants, 396 could be identified as having been implanted using either a RW or eRW approach and 39 as implanted using a CS approach [4, 18, 20, 21, 23, 24]. The translocation risk, extrapolated from these studies, was $3 \%$ (12/396) for the eRW/RW approach and 23\% (9/39) for the CS approach. Examination of the translocation rates in the studies that additionally discerned between eRW and RW approaches revealed a slightly lower average translocation rate for eRW insertions $(0.67 \%, 1 / 150$ vs. $3.5 \%, 6 / 170$, respectively) $[18,20,21,23]$. Therefore, with regards to achieving an optimal scalar position, round window insertion seems the best choice for SME-implantations. Furthermore, extending the round window opening by drilling away the crista fenestrae may result in an even better orientation of the sheath during insertion, with a more direct trajectory down the basal turn and subsequent lower translocation rate [23].

Moreover, our results further confirm that this in turn gives rise to excellent levels of speech perception with this particular electrode. We found the CVC-phoneme score to improve from $27.1 \%$ preoperatively to $72.1 \%$ at 2 months and $82.1 \%$ at 12 months post-operatively. These scores are comparable to, if not better than, what is reported in other studies [19, 21, 23, 25]. Shaul et al. studied 18 patients with similar levels of preoperative phoneme scores implanted with a SME using the eRW approach [26]. They reported an trode array located in the scala tympani (ST) and those with a translocated electrode array [4] 
membrane over time. Elliot et al. modelled an estimated 100 -fold increase in RW membrane stiffness after cochlear implantation, which was predicted to lead to $20 \mathrm{~dB}$ hearing loss across frequencies below $1000 \mathrm{~Hz}$ [27]. Furthermore, a study by Rowe et al. investigating residual hearing loss for different routes of electrode insertion using a Guinea pig model showed a pronounced delayed, low frequency hearing loss in round window insertions, which was not observed after cochleostomy insertions [28]. The authors speculate that this effect is the result of post-insertion packing of the round window opening with a soft tissue graft, and subsequent fibrotic and neo-osteogenic changes leading to reduced RW membrane compliance. Other possible reasons for lower RHP rates in eRW insertions are extensive drilling of the so-called 'hook-region', with subsequent possible acoustic trauma, disruption of the vascular drainage and blockage of the endolymphatic system due to fibrous tissue formation [29].

Besides insertions via the extended round window- or cochleostomy approaches, SME insertions via so-called 'pure round window approach' and 'round window enlargement' have also been described, which do not require drilling the crista fenestrae [18, 21, 30]. Although in essence less traumatic than the other approaches, we believe the pure round window approach is only possible in patients with a favorable temporal bone anatomy and even in these cases, insertion of the plastic sheath that houses the electrode is often only possible after extensive drilling of the superior lip of the round window niche (round window enlargement) or carefully drilling away the crista fenestrae (extended round window). In our experience, and as also described by Shaul et al., insertion of the sheath in patients with an inadequately extended round window opening can lead to the deformation and compression of the sheath-opening [23]. Attempting to advance the array out of the compressed sheath-opening could lead to protrusion of the electrode through the side of the sheath. The silicone cochleostomy sizer tool which is supplied with the implant is, therefore, a valuable asset in determining whether an adequate access has been achieved, especially when the access to the scala tympani is challenging. Liebscher et al. described the scalar position and hearing outcomes of 156 patients implanted with the SME, 127 of whom via the round window, but did not elaborate on whether extensive drilling of the bony overhang preceded electrode insertion [21]. In the subset of patients for whom speech perception testing was performed, they were able to achieve a median speech recognition score of $75 \%(n=148$, range: $27.5-95 \%$ ) using the Freiburg monosyllable word test. However, the fact that so little studies report on 'pure round window' insertions or do not exactly describe the procedure leading up to insertion of the electrode emphasizes the exceptionality of the anatomical circumstances necessary to insert the SME directly through the round window 
membrane. On the other hand, so-called 'round window enlargement', in which the posterosuperior lip of the bony overhang covering the round window niche is extensively drilled without additional intracochlear drilling, seems to deliver good results, as described by Ramos et al. and IsoMustajärvi et al., who reported on 18 SME insertions via a 'round window membrane' approach, but add to their description of surgical technique that the superior bony overhang of the round window niche was largely drilled away to achieve an optimal view of the round window membrane $[20,22]$. Their technique may be comparable to what other authors describe as round window enlargement and resulted in residual hearing preservation $\left(\mathrm{PTA}_{125-500}<80 \mathrm{~dB}\right)$ in $14 / 17$ ears (82\%).

In summary, each of the surgical techniques has its advantages and limitations. To achieve correct positioning of the SME with the highest chances of ST placement, the different round window techniques seem to be favored over the cochleostomy technique. Between the insertions through the round window, the pure round window approach would be the technique of choice if the individual anatomy and silicone sheath would allow it. However, in the majority of the cases insertion directly through the RW is not possible and for those cases, round window enlargement or round window extension will also provide excellent ST positioning and speech perception scores. On the other hand, the extended round window approach showed suboptimal results for preservation of residual hearing in this study, with a level of loss comparable to that of patients implanted via cochleostomy with translocation to the SV [4]. The best results specifically regarding residual hearing preservation seem to have been reported with the cochleostomy technique if the electrode does not translocate and is positioned entirely within the scala tympani $[4,22]$. To accomplish this, one should focus on placing the cochleostomy as inferiorly as possible, to direct the sheath away from the spiral lamina in the direction of the floor of the scala tympani and minimize the risk of cochleostomy-associated translocation [31]. However, a great anatomical variation exists between individuals and, therefore, the risk of direct translocation to the SV should always be considered when using the cochleostomy approach.

The main limitation of the current study is that the number of participants is relatively small. The sample size was chosen to be able to compare to our previous study, in which we investigated CI outcomes using the SME implanted via cochleostomy. However, the direct comparison to this previous study was hampered by differences between the two patient populations, as the current study was conducted solely in participants with post-lingual hearing loss, with better preoperative pure tone audiometry thresholds and speech perception, and the surgery was conducted by three different surgeons. Additionally, residual hearing has been found to deteriorate beyond 24 months after surgery [32]. Therefore, the residual hearing loss observed in our study population may still proceed past the last follow-up timepoint. Lastly, the terminology used to describe the exact approach taken to access the scala tympani was found to differ in other studies, also complicating direct comparison; although several papers report on using the round window approach it is unclear whether this includes complete or partial removal of the bony overhang, or drilling of the crista fenestrae. Therefore, further studies investigating the effect of the different surgical approaches on CI outcome using this electrode are necessary.

\section{Conclusion}

In this prospective study, we showed that correct positioning of the SME within the scala tympani can consistently be achieved using the extended round window approach, resulting in excellent levels of speech perception. However, the results also indicate that extending the round window opening can be suboptimal for the residual acoustic hearing, emphasizing that with this particular electrode there is no 'all-purpose' insertion approach for cochlear implantation. Where the anatomy is favorable, an insertion via the round window should be considered; if surgically feasible, patients with residual hearing should be implanted via 'pure round window' or 'round window enlargement' approach, whilst patients without residual hearing should be implanted via an extended round window approach. In cases where the anatomy is not favorable, i.e. in patients in whom round window insertions are not possible due to inadequate visibility of the RW membrane, an inferiorly placed cochleostomy may offer a solution and result in good levels of both residual hearing preservation and speech perception if the electrode is placed within the scala tympani.

Funding This study was financialy supported by Cochlear Ltd.

Open Access This article is licensed under a Creative Commons Attribution 4.0 International License, which permits use, sharing, adaptation, distribution and reproduction in any medium or format, as long as you give appropriate credit to the original author(s) and the source, provide a link to the Creative Commons licence, and indicate if changes were made. The images or other third party material in this article are included in the article's Creative Commons licence, unless indicated otherwise in a credit line to the material. If material is not included in the article's Creative Commons licence and your intended use is not permitted by statutory regulation or exceeds the permitted use, you will need to obtain permission directly from the copyright holder. To view a copy of this licence, visit http://creativecommons.org/licenses/by/4.0/. 


\section{References}

1. O'Connell BP, Hunter JB, Wanna GB (2016) The importance of electrode location in cochlear implantation. Laryngoscope Investig Otolaryngol 1(6):169-174

2. Wanna GB et al (2014) Impact of electrode design and surgical approach on scalar location and cochlear implant outcomes. Laryngoscope 124(Suppl 6):S1-7

3. Nadol JB Jr et al (2001) Histopathology of cochlear implants in humans. Ann Otol Rhinol Laryngol 110(9):883-891

4. Heutink F et al (2020) The evaluation of a slim perimodiolar electrode: surgical technique in relation to intracochlear position and cochlear implant outcomes. Eur Arch Otorhinolaryngol 277(2):343-350

5. Holden LK et al (2013) Factors affecting open-set word recognition in adults with cochlear implants. Ear Hear 34(3):342-360

6. Aschendorff A et al (2007) Quality control after insertion of the nucleus contour and contour advance electrode in adults. Ear Hear 28(2 Suppl):75s-79s

7. Finley CC et al (2008) Role of electrode placement as a contributor to variability in cochlear implant outcomes. Otol Neurotol 29(7):920-928

8. Berg KA et al (2019) Speech recognition as a function of the number of channels in perimodiolar electrode recipients. J Acoust Soc Am 145(3): 1556

9. Hughes ML, Abbas PJ (2006) Electrophysiologic channel interaction, electrode pitch ranking, and behavioral threshold in straight versus perimodiolar cochlear implant electrode arrays. J Acoust Soc Am 119(3):1538-1547

10. Hughes ML, Stille LJ (2010) Effect of stimulus and recording parameters on spatial spread of excitation and masking patterns obtained with the electrically evoked compound action potential in cochlear implants. Ear Hear 31(5):679-692

11. Mens LH, Boyle PJ, Mulder JJ (2003) The Clarion Electrode positioner: approximation to the medial wall and current focussing? Audiol Neurootol 8(3):166-175

12. Wanna GB et al (2015) Impact of intrascalar electrode location, electrode type, and angular insertion depth on residual hearing in cochlear implant patients: preliminary results. Otol Neurotol 36(8):1343-1348

13. Ltd., C. Cochlear Nucleus CI532: https://www.cochlear.com/uk/ ci532 (April 2019)

14. Jiam NT, Limb CJ (2016) The impact of round window vs cochleostomy surgical approaches on interscalar excursions in the cochlea: Preliminary results from a flat-panel computed tomography study. World J Otorhinolaryngol Head Neck Surg 2(3):142-147

15. World Medical Association Declaration of Helsinki (2013) ethical principles for medical research involving human subjects. JAMA 310(20):2191-2194

16. Skarzynski H et al (2013) Towards a consensus on a hearing preservation classification system. Acta Otolaryngol Suppl 564:3-13

17. Snels CWM et al (2020) Short and long term preservation of hearing thresholds corrected for natural hearing loss in cochlear implant recipients using a straight electrode. Cochlear Implants Int 21(2):110-116

18. Aschendorff A et al (2017) Clinical investigation of the Nucleus Slim Modiolar Electrode. Audiol Neurootol 22(3):169-179

19. Durakovic $\mathrm{N}$ et al (2020) Immediate and 1-year outcomes with a slim modiolar cochlear implant electrode array. Otolaryngol Head Neck Surg 162(5):731-736

20. Iso-Mustajarvi M et al (2020) Preservation of residual hearing after cochlear implant surgery with slim modiolar electrode. Eur Arch Otorhinolaryngol 277(2):367-375

21. Liebscher $\mathrm{T}$ et al (2021) Electrode translocations in perimodiolar cochlear implant electrodes: audiological and electrophysiological outcome. Z Med Phys 31(3):265-275

22. Ramos-Macias A et al (2017) Hearing preservation with the slim modiolar electrode nucleus CI532(R) cochlear implant: a preliminary experience. Audiol Neurootol 22(6):317-325

23. Shaul C et al (2020) Slim, modiolar cochlear implant electrode: melbourne experience and comparison with the contour perimodiolar electrode. Otol Neurotol 41(5):639-643

24. Nassiri AM et al (2020) Hearing preservation outcomes using a precurved electrode array inserted with an external sheath. Otol Neurotol 41(1):33-38

25. Holder JT et al (2019) Matched cohort comparison indicates superiority of precurved electrode arrays. Otol Neurotol 40(9):1160-1166

26. Shaul C et al (2018) Scalar localisation of peri-modiolar electrodes and speech perception outcomes. J Laryngol Otol 132(11):1000-1006

27. Elliott SJ, Ni G, Verschuur CA (2016) Modelling the effect of round window stiffness on residual hearing after cochlear implantation. Hear Res 341:155-167

28. Rowe D et al (2016) Delayed low frequency hearing loss caused by cochlear implantation interventions via the round window but not cochleostomy. Hear Res 333:49-57

29. Richard C et al (2012) Round window versus cochleostomy technique in cochlear implantation: histologic findings. Otol Neurotol 33(7):1181-1187

30. Iso-Mustajarvi M et al (2020) Preservation of residual hearing after cochlear implant surgery with slim modiolar electrode. Eur Arch Otorhinolaryngol 277(2):367-375

31. Atturo F, Barbara M, Rask-Andersen H (2014) Is the human round window really round? An anatomic study with surgical implications. Otol Neurotol 35(8):1354-1360

32. Helbig $S$ et al (2016) Long-term hearing preservation outcomes after cochlear implantation for electric-acoustic stimulation. Otol Neurotol 37(9):e353-e359

Publisher's Note Springer Nature remains neutral with regard to jurisdictional claims in published maps and institutional affiliations. 